\title{
Study on Smart Growth Metric Model
}

\author{
Xiaolong $\mathrm{Xu}^{1, \mathrm{a}}$ \\ ${ }^{1}$ North China Electric Power University(Baoding), Baoding 071000, China \\ a5864712@qq.com
}

\begin{abstract}
We establish the metric model, Smart Growth Metric (SGM). It is divided into Economic Status Metric system, Social Status Metric system and Ecological Environment Metric system. Each system consists of several indicators. Based on the ten principles of smart growth and $3 \mathrm{E}$ 's, we select the indicators, such as urban per capita road area and the degree of land mixing. We make some adjustments to these indicators to evaluate the economy, society and environment. By Gray Relational Analysis, we assign weights to each indicator in their system. Since different cities are at different levels, we give the economy, society and environment different weight combinations. These combinations apply to underdeveloped cities, developing cities and developed cities, respectively.
\end{abstract}

Keywords: Gray Relational Analysis, Gray Forecasting Model, Smart Growth

\section{Introduction}

Urbanization is the main theme of the development of human society. However, the resources are limited. The countries and people in the world are confronted with such problems as population expansion, shortage of resources, serious environmental pollution, traffic congestion and increasing public security risks. Therefore, the realization of sustainable urban development is one of the most important research topics in the world today.

In order to realize the sustainable development of the city, many countries and regions have implemented the smart growth plan for a modern city with prosperous economy, social fairness and sustainable ecological environment.

Reasonable smart growth plan allows the city to achieve a rational distribution of resources, fair social competition and increase people's well-being index. However, simply raising one or a few of these indicators will make urban development rigid. Meanwhile, the economy, society and ecology environment cannot develop in a balanced and healthy way.

\section{Construction of ESM}

ESM is the foundation of a city. The government has implemented many policies in order to take care of the economic development. The better economic environment, the better development. Therefore, the urban economy has a great impact on the sustainable development of a city. Based on the principles, we divide the ESM into the following indicators: (1) Urban per capita GDP(UGDP);(2) Industry level(IL); (3) Average annual per capita income(AI);(4) Average annual per capita consumption(AC)

Then we develop a Economic Status Metric (ESM) based on the linear combination of four indicators created by UGDP, IL, AI and AC. The specific process is as follows:

First, we should make all indicators normalization:

$$
S_{i}=\frac{\left(U_{i}-L_{i}\right)\left(X_{i}-T_{i}\right)}{\left(U_{i}+L_{i}-2 T_{i}\right) X_{i}+U_{i} T_{i}+L_{i} T_{i}-2 U_{i} L_{i}}
$$

$X_{i}$ is the actual value of indicator $\mathrm{i}, U_{i}$ is the upper limit of indicator $\mathrm{i}, L_{i}$ is the lower limit of indicator $\mathrm{i}$, and $T_{i}$ is the critical threshold of indicator $\mathrm{i}$. 
Then, based on the Grey Relational Analysis, we calculate the weight of the four indicators on ESM, to give the equation (2):

$$
E S M=\lambda_{1} U G D P+\lambda_{2} I L+\lambda_{3} A I+\lambda_{3} A C
$$

Where $\lambda_{1}, \lambda_{2}$ and $\lambda_{3}$ are weights of four indicators, and their sum equals to 1 . We conclude that the larger ESM, the better Economic Status.

\section{Construction of SSM}

SSM is the embodiment of daily life, and is closely related with the masses. Good social status will enhance a city mental outlook and material environment, optimize the overall lifestyle of residents and the choice of values, and enhance the residents of the city's identity, pride, sense of superiority. These will be transformed into the city cohesion and inspiration, in order to achieve the ultimate goal of continuous improvement of the city comprehensive competitiveness and promote the healthy sustainable development of cities. Based on the principles, we divide the SSM into the following indicators: (1) Total value of urban cultural industry(TVI); (2) Urban per capita road area(URA);(3) Gini coefficient(G) ;(4) Per capita housing area(HA);(5) The average number of years of education(YE); (6) The degree of land mixing(LM).

Similarly, according to 2 , we can get the equation:

$$
S S M=\lambda_{1} T V I+\lambda_{2} U R A+\lambda_{3}(1-G)+\lambda_{4} H A+\lambda_{5} Y E+\lambda_{6} L M
$$

Because $\mathrm{G}$ is negative correlation with SSM, we use $1-\mathrm{G}$ in the equation (3).

\section{Construction of EEM}

EEM is closely related to the development of the city. The development of the city will inevitably lead to changes in the environment, the expansion of the region, environmental pollution and changes in green area. We must pay attention to these issues. In the process of urban development, we should make every effort to ensure the stability of the ecological environment, so as to ensure the sustainable development of the city. Based on the principles, we divide the EEM into the following indicators: (1) Per capita public green area(GA) ;(2) Electricity consumption per capita annual(EC) ;(3) Overall pollution degree of city(OP).

Similarly, according to 2, we can get the equation:

$$
E E M=\lambda_{1} G A+\lambda_{2}(1-E C)+\lambda_{3}(1-O P)
$$

Because EC and OP is negative correlation with EEM, we use 1-EC and 1-OP in the equation

\section{Construction of SGM}

Based on the above analysis, we get the expression that affects ESM, SSM and EEM. But the correlation between ESM, SSM and EEM is also uncertain. So we use GRA to build our Smart Growth Metric(SGM). And we define the SGM's equation as follows:

$$
S G M=\alpha E S M+\beta S S M+\gamma E E M
$$

Where $\alpha, \beta$ and $\gamma$ are weights of three systems.

We refer to the statistical yearbook, bulletin and national and international indicators of the corresponding standards to determine the threshold, upper and lower limits. Then we formulate four-level grading standards. 
Table 1: Smart Growth Metric grading standards

\begin{tabular}{ccc}
\hline Grade & Value & Qualitative evaluation \\
\hline I & $>0.75$ & Excellent \\
II & $0.50 \sim 0.75$ & Good \\
III & $0.25 \sim 0.50$ & Moderate \\
IV & $<0.25$ & Bad \\
\hline
\end{tabular}

\section{Verification}

According to the current development status of Zurich and Beira, we selected the growth plan. In the process above, we obtain the indicators of each city. We normalize these indicators. We first provide the weights of the 4 indicators in ESM model, 6 indicators in SSM model and 3 indicators in EEM model, all calculated by the Grey Relational Analysis in Table 2.

Table 2: The Weight of Indicators

\begin{tabular}{cccccc}
\hline ESM & Weight & SSM & Weight & EEM & Weight \\
\hline UGDP & 0.3428 & TVI & 0.1579 & GA & 0.3418 \\
IL & 0.2574 & URA & 0.0785 & EC & 0.2926 \\
AI & 0.1957 & G & 0.2617 & OP & 0.3856 \\
AC & 0.2041 & HA & 0.1154 & & \\
& & YE & 0.1637 & & \\
& & LM & 0.2228 & & \\
\hline
\end{tabular}

We use Full Permutation Polygon Synthesis Illustration method to visualize the effect of different indicators on ESM SSM EEM. We show it as follows:
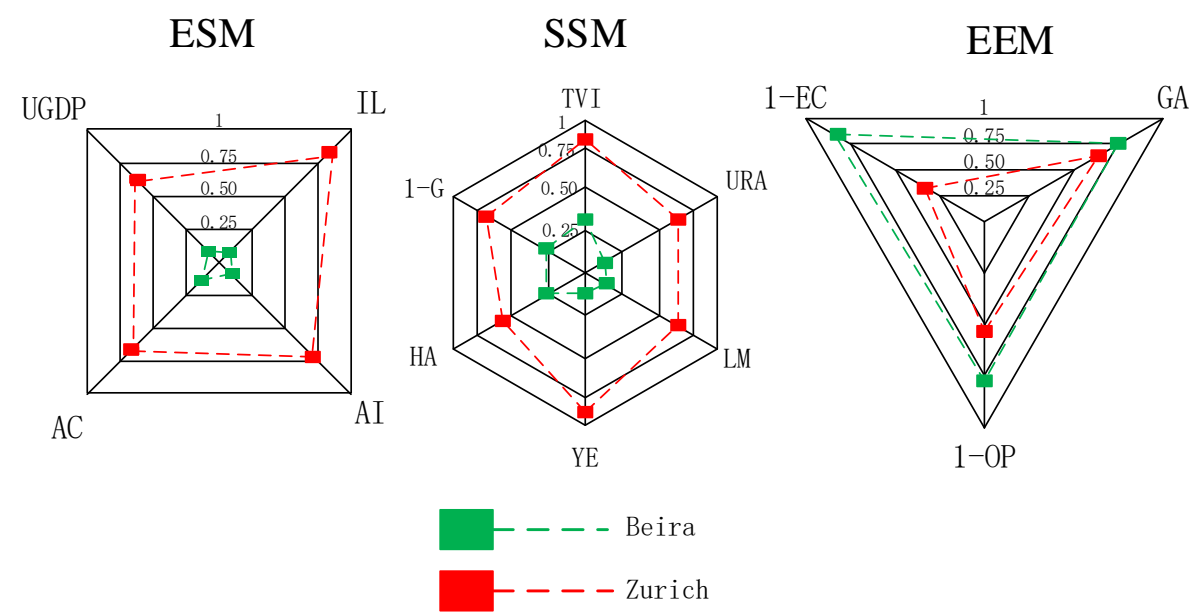

Figure 1: Indicators distribution

Similarly, we visualize the effect of ESM SSM EEM on SGM. We show it as follows: 


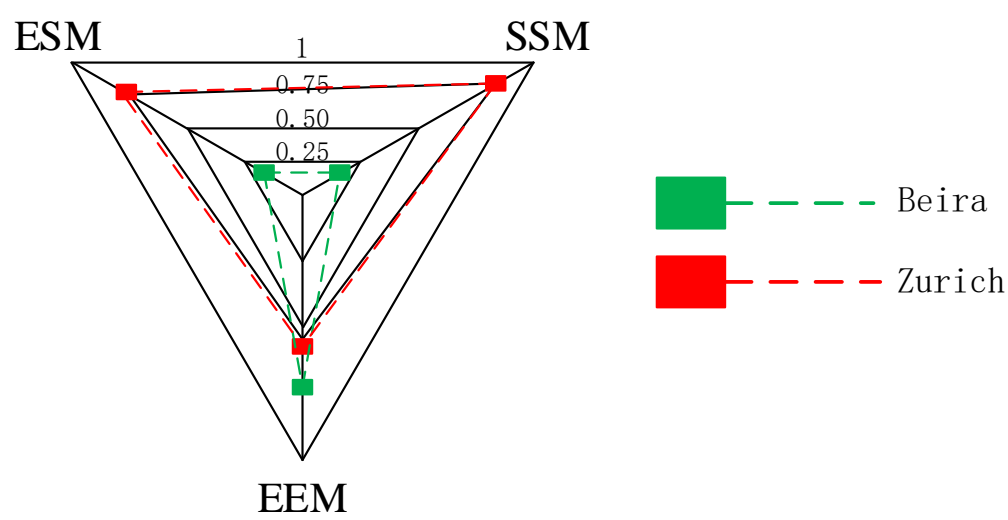

Figure 2: Systems distribution

Then, we select ESM SSM EEM weights according to whether the city is a developed city. Finally, according to the SGM and grading standards to determine whether the current growth plan is successful. The equations are as follows:

$$
\text { Zurich( Switzerland): } \quad S G M=0.3 E S+0.3 S S+0.4 E E=0.7408
$$

The growth plan is good.

Beira(Mozambique): $\quad S G M=0.4 E S+0.4 S S+0.2 E E=0.2647$

The growth plan is Moderate.

\section{Conclusion}

First of all, we analyze Zurich. Through the various indicators of Zurich, we can see that Zurich, whether economic, social, environmental approach perfection. Its SGM is 0.75 or so, that expresses the growth plan achieve success and the level of sustainable development is excellent.

Then analyze Beira. Compared to Zurich, Beira's indicators seem terrible, only the indicators in the ecological environment is better. Economic status and social status are the main reasons for its low SGM. Growth plans can be wandered on the brink of failure, and the level of sustainability is very poor.

\section{References}

[1] https://en.wikipedia.org/wiki/Gross_domestic_product

[2] Sun X, Liu X S, Li F, Tao Y. Comprehensive evaluation of sustainable development for different scale cities in China. Acta Ecological Sinica,2016,36( 17) : 5590-5600.

[3] S B Zhou, W T Qi, A M Du, H He. A method to evaluate coordination between regional economic, social development and water resources[J]. IOP Conference Series: Earth and Environmental Science,2016,39(1).

[4] Li F, Liu X S, Hu D and Wang R S 2007 Evaluation method and its application for urban sustainable development Acta Ecological Sinica11:4793-802.

[5] http://baike.baidu.com/item/\%E5\%9F\%8E\%E5\%B8\%82\%E6\%96\%87\%E5\%8C\%96/10848136

[6] https://en.wikipedia.org/wiki/Gini_coefficient

[7] Dr. Mohan Kumar Pradhan. Multi-Objective Optimization of MRR, TWR and Radial Overcut of EDMed AISI D2 Tool Steel Using Response Surface Methodology, Grey Relational Analysis And Entropy Measurement[J]. J. Manuf. Sci. Prod.,2012,12(1).

[8] Y.Z. Wang, J. Zhao, Y. Wang, Q.S. An. Multi-objective optimization and grey relational analysis on configurations of organic Rankine cycle[J]. Applied Thermal Engineering,2016. 